\title{
Versatility of a hard X-ray Kirkpatrick-Baez focus characterized by ptychography
}

\section{Klaus Giewekemeyer, Robin N. Wilke, Markus Osterhoff, Matthias Bartels, Sebastian Kalbfleisch and Tim Salditt}

J. Synchrotron Rad. (2013). 20, 490-497

\section{IUCr Journals CRYSTALLOGRAPHY JOURNALS ONLINE}

Copyright (C) International Union of Crystallography

Author(s) of this paper may load this reprint on their own web site or institutional repository provided that this cover page is retained. Republication of this article or its storage in electronic databases other than as specified above is not permitted without prior permission in writing from the IUCr.

For further information see http://journals.iucr.org/services/authorrights.html 
Journal of

Synchrotron

Radiation

ISSN 0909-0495

Received 3 December 2012

Accepted 25 February 2013

\section{Versatility of a hard X-ray Kirkpatrick-Baez focus characterized by ptychography}

\author{
Klaus Giewekemeyer, ${ }^{\mathrm{a}, \mathrm{b} *}$ Robin N. Wilke, ${ }^{\mathrm{a}}$ Markus Osterhoff, ${ }^{\mathrm{a}}$ Matthias Bartels, ${ }^{\mathrm{a}}$ \\ Sebastian Kalbfleisch ${ }^{\mathrm{a}}$ and Tim Salditt ${ }^{\mathrm{a}}$
}

alnstitut für Röntgenphysik, Georg-August-Universität Göttingen, 37077 Göttingen, Germany, and

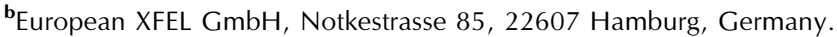

E-mail: klaus.giewekemeyer@xfel.eu

\begin{abstract}
In the past decade Kirkpatrick-Baez (KB) mirrors have been established as powerful focusing systems in hard X-ray microscopy applications. Here a ptychographic characterization of the KB focus in the dedicated nano-imaging setup GINIX (Göttingen Instrument for Nano-Imaging with X-rays) at the P10 coherence beamline of the PETRA III synchrotron at HASLYLAB/DESY, Germany, is reported. More specifically, it is shown how aberrations in the KB beam, caused by imperfections in the height profile of the focusing mirrors, can be eliminated using a pinhole as a spatial filter near the focal plane. A combination of different pinhole sizes and illumination conditions of the $\mathrm{KB}$ setup makes the prepared optical setup well suited not only for high-resolution ptychographic coherent X-ray diffractive imaging but also for moderateresolution/large-field-of-view propagation imaging in the divergent KB beam.
\end{abstract}

C 2013 International Union of Crystallography

Printed in Singapore - all rights reserved

\section{Introduction}

In recent years Kirkpatrick-Baez (KB) mirrors, among other focusing techniques, have been established as a powerful and efficient way to focus hard X-ray beams for imaging at the nanoscale (Robinson et al., 2003; Sakdinawat \& Attwood, 2010), with beam sizes below $50 \mathrm{~nm}$ in two dimensions (Matsuyama et al., 2006) and $10 \mathrm{~nm}$ in one dimension (Mimura et al., 2010). The high efficiency of KB mirrors, together with their grazing-incidence geometry, makes them attractive focusing devices generating very high focal fluences for both synchrotron (Hignette et al., 2005; Matsuyama et al., 2006) and $\mathrm{X}$-ray free-electron laser (FEL) sources (Mimura et al., 2008; Boutet \& Williams, 2010; Siewert et al., 2012). By coherent illumination of reflective focusing optics with a monochromatic coherent synchrotron beam, either through long propagation between the monochromator and the mirror (Takahashi et al., 2010) or by selecting a coherent fraction of the incoming beam with apertures (Kewish et al., 2010a), the resulting focus can be made fully coherent and thus applicable for coherent X-ray diffractive imaging (CDI).

Up to now a wealth of different implementations of CDI have been developed, each with their own specific requirements and advantages (Quiney, 2010; Nugent, 2010; Thibault \& Elser, 2010). Plane-wave CDI, for example, as one of the original forms of CDI (Miao et al., 1999; Chapman et al., $2006 b$ ), requires a well prepared planar wavefront that completely illuminates an isolated sample in the center. Whereas this geometry may seem rather restrictive from a general perspective, it can be naturally implemented in the case of samples injected into a FEL beam (Seibert et al., 2011; Chapman et al., 2011). Here the specimens are investigated by single pulses per sample in a 'diffract and destroy' manner (Chapman et al., 2006a). On the other hand, a plane-wave illumination introduces possible ambiguities in the reconstruction (Miao et al., 1998), which are not present if the sample is placed into the spherical wavefront of a divergent beam (Quiney et al., 2005; Williams et al., 2006). In the latter imaging modality a near-field diffraction pattern (an in-line hologram) of the sample is produced at the detector. Just as in the production of a planar wavefront for plane-wave CDI, an experimental challenge is given here by the demand of a clean spherical wavefront similar to an ideal point source. If aberrations cannot be sufficiently minimized, an accurate knowledge of the complex illumination has to be obtained in an independent measurement in order to determine the accurate complex sample exit wave (Williams et al., 2006; Quiney et al., 2005).

Going beyond single-image CDI schemes, it has been shown in recent years that adding diversity by translating the sample laterally (Rodenburg et al., 2007; Thibault et al., 2008) or longitudinally (Putkunz et al., 2011) through a well confined coherent illuminating wavefield can greatly improve the convergence rates of iterative reconstruction schemes. In the most popular approach, named ptychographic CDI (PCDI) (Rodenburg et al., 2007; Thibault et al., 2008; Guizar-Sicairos \& Fienup, 2008; Rodenburg, 2008), a sample is scanned on a grid perpendicular to the optical axis, so that neighboring 
illuminated areas on the sample exhibit a large overlap which in turn leads to strong constraints improving the reconstruction. Here the demands and the required pre-knowledge on the illuminating wavefield are much more relaxed than in the single-image techniques. For example, the lateral extension of the sample may be much larger than the size of the illumination which itself may also have considerable aberrations from an ideal spherical or planar wavefront. This allows for a certain freedom to optimize the illumination in terms of size (and thus scanning time) and fluence on the sample (and thus dose and resolution).

Here we show how the coherent focus of a hard X-ray KB system can be tuned for different imaging modalities and thus provide an example illustrating the versatility of this focusing device. Furthermore, we explore the use of a pinhole near the focal plane as a spatial filter for the far-field diffraction pattern. For characterization of the complex wavefield in different modalities we have used PCDI, nowadays a well established technique for characterizing high-resolution X-ray optics (Kewish et al., 2010a,b; Schropp et al., 2010; GuizarSicairos et al., 2010, 2011; Mastropietro et al., 2011; Hönig et al., 2011; Takahashi et al., 2011; Wilke et al., 2012; Huang et al., 2012). It is noted that it has been shown recently for zoneplate focusing optics (Guizar-Sicairos et al., 2012) that a sharp confinement of the illumination near the sample improves the image quality in PCDI.

\section{Experiment}

The experiment (for a schematic see Fig. 1) was performed at the P10 coherence beamline of the PETRA III synchrotron source at DESY in Hamburg, Germany, using the Göttingen Instrument for Nano-Imaging with X-rays (GINIX) (Kalbfleisch et al., 2011; Kalbfleisch, 2012). This modular device is mounted on a single optical table and can be moved out and into the optical axis, aligned and made ready to use within less than a day. As a central component the device comprises a KB focusing system which provides a hard X-ray nano-focus (Salditt et al., 2011; Kalbfleisch et al., 2011). It can be used for propagation-based imaging applications, either directly (Olendrowitz et al., 2012) or after coupling into further confining optics [e.g. waveguides (Bartels et al., 2012; Krüger et al., 2012; Giewekemeyer et al., 2011b, using a similar KB setup)].

In the current experiment (see Fig. 1) the undulator beam was monochromatized to a photon energy of $7.9 \mathrm{keV}$ and confined by two pairs of hybrid metal single-crystal blades
(Xenocs, France), oriented in the horizontal and vertical direction (see slits S1H and S1V in Fig. 1), before entering the system of the two KB mirrors (see MV and MH in Fig. 1).

The KB mirror system that was used consists of two elliptical mirrors with a fixed shape (Kalbfleisch et al., 2011; Kalbfleisch, 2012). The horizontally focusing mirror (4.05 mrad incidence angle, $200 \mathrm{~mm}$ focal length) was polished by Elastic Emission Machining (JTEC, Japan) (Mimura et al., 2007) while the vertically focusing mirror (3.954 mrad incidence angle, $302 \mathrm{~mm}$ focal length) was fabricated by Magnetorheological Finishing (WinlightX, France) (Harris, 2011). To increase the reflectivity at high photon energies both mirrors were coated with a thin Pd layer (respective thicknesses $35 \mathrm{~nm}$ and $55 \mathrm{~nm}$ ). The final deviations (peak-to-valley) from the ideal elliptical shape, as obtained by optical metrology, were $4.8 \mathrm{~nm}$ and $13.9 \mathrm{~nm}$, respectively. To reduce beam-induced degradation, the KB setup is operated in an ultrahigh-vacuum vessel with silicon nitride membranes (Silson, UK) used as window materials. Simulations of the focal field based on the measured height profiles of both mirrors were presented by Salditt et al. (2011).

For the ptychographic scanning experiments (see below) a Pilatus $300 \mathrm{~K}$ detector (Dectris, Switzerland) was used which was placed $5.49 \mathrm{~m}$ downstream of the focal plane.

To block unfocused and partly focused components of the beam exiting the KB system a pinhole large enough (diameter $=1 \mathrm{~mm}, 450 \mu \mathrm{m}$-thick $\mathrm{W})$ not to cut the tails of the focused beam component was placed downstream of the KB exit window. 

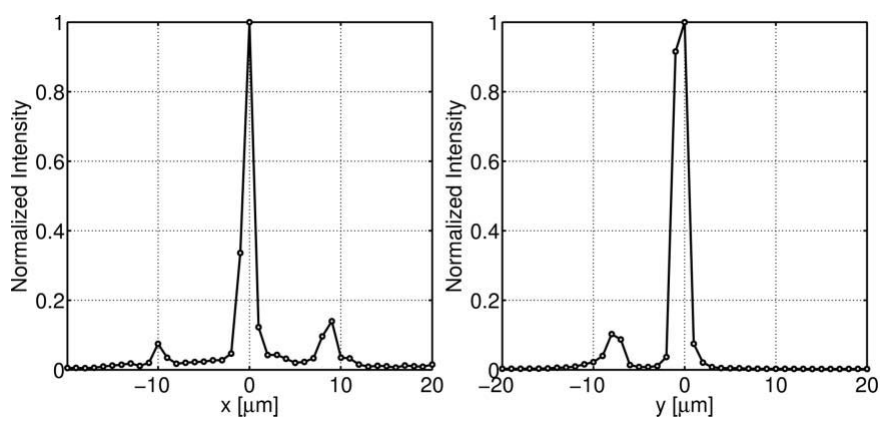

Figure 2

Intensity scan of a small pinhole $(1.4 \mu \mathrm{m}$ in diameter) through the focal plane of the KB system. The detector was a photodiode. Prominent side lobes are visible in both the horizontal and vertical directions. Note that the slits S1V, S1H and S2H were all set to a width of $100 \mu \mathrm{m}$ during the scans, i.e. the incoming beam was considerably confined before entering the KB system.

As it becomes evident by scanning a small pinhole through the KB focus in a direction perpendicular to the beam, both horizontally and vertically, the focus exhibited rather prominent side lobes at a distance of 7-10 $\mu \mathrm{m}$ away from the beam center (see Fig. 2). The side lobes are attributed to refraction of the primary beam from the beveled edges of the singlecrystal slits ( $\mathrm{Li}$ et al., 2008) which were used to reduce the amount of unwanted scattering contributions in the far field of the KB (Osterhoff, 2011). ${ }^{1}$ With a detector distance of $5.49 \mathrm{~m}$ and a detector pixel size of $172 \mu \mathrm{m}$ this does not allow for sufficient sampling of the corresponding far-field diffraction pattern in order to reconstruct the focus and its side lobes by coherent diffractive imaging methods.

The side lobes could not be sufficiently suppressed by adding a standard slit (jj-X-ray, Denmark; see S2H in Fig. 1) upstream of the $\mathrm{KB}$ system (see $\mathrm{S} 2 \mathrm{H}$ in Fig. 1). In a first experiment a Pt pinhole (Plano, Germany; polished with a focused ion beam) of $200 \mu \mathrm{m}$ thickness and $\sim 8 \mu \mathrm{m}$ diameter was therefore placed $3.95 \mathrm{~mm}$ upstream of the focal plane. Its longitudinal position was determined with two on-axis visuallight microscopes, one upstream and one downstream of the focal plane.

Next, a Ta test sample (ATN/XRESO-50HC; NTT-AT, Japan), fabricated by nanolithography, with a structure depth of $500 \mathrm{~nm}$ was inserted into the focal plane. A ptychographic scan on a Cartesian grid with $200 \mathrm{~nm}$ step size and 21 scan positions, both in the horizontal and vertical direction perpendicular to the optical axis, was performed using an illumination time of $50 \mathrm{~ms}$ per step, defined by a fast shutter. For the scan the gap sizes of the slits $\mathrm{S} 1 \mathrm{~V}, \mathrm{~S} 1 \mathrm{H}$ and $\mathrm{S} 2 \mathrm{H}$ were set to $200 \mu \mathrm{m}, 100 \mu \mathrm{m}$ and $500 \mu \mathrm{m}$, respectively (for an overview of the different scan settings, see Table 1). Note that the beam was attenuated by a factor of the order of $4 \times 10^{-3}$ in order not to exceed the maximum measurable flux using the Pilatus detector $\left[\gtrsim 2 \times 10^{6}\right.$ photons s $^{-1}$ pixel $^{-1}$ (Toyokawa et al., 2010)].

\footnotetext{
${ }^{1}$ Note that the distance of the side lobes to the optical axis is larger here than described and simulated by Osterhoff (2011). This may be explained by the lower photon energy used in the present experiment as well as the simplified model of the slits that was used.
}

Table 1

Main optical settings of the considered different ptychographic datasets.

For explanation of the nomenclature, see Fig. 1.

\begin{tabular}{|c|c|c|c|c|c|}
\hline \multirow[b]{2}{*}{ Dataset } & \multicolumn{3}{|c|}{ Slit gap sizes (mm) } & \multirow{2}{*}{$\begin{array}{l}\text { Pinhole } \\
\text { diameter }(\mu \mathrm{m})\end{array}$} & \multirow[b]{2}{*}{$z_{\mathrm{P} 2}(\mathrm{~mm}$} \\
\hline & S1V & $\mathrm{S} 1 \mathrm{H}$ & $\mathrm{S} 2 \mathrm{H}$ & & \\
\hline 1 & 0.2 & 0.1 & 0.5 & 8 & 3.95 \\
\hline 2 & 0.1 & 0.1 & 0.5 & 8 & 3.95 \\
\hline 3 & 0.1 & 0.1 & 0.1 & 1.4 & 0.5 \\
\hline
\end{tabular}

In another ptychographic scan the gap of S1V was closed to $100 \mu \mathrm{m}$, leaving all remaining gap widths the same. A ptychographic scan with a step size of $200 \mathrm{~nm}$ and $51 \times 51$ positions was then performed using an illumination time of $1 \mathrm{~s}$ per step. For this scan the beam was attenuated by a total factor of $\sim 5.6 \times 10^{-5}$. A section of $21 \times 21$ positions was selected from the whole dataset because of positioning drift accumulated over the course of the whole scan comprising 2601 positions.

Finally, the pinhole P2 used above was replaced by a smaller pinhole of diameter $1.4 \mu \mathrm{m}$, milled into $200 \mu \mathrm{m} \mathrm{W}$ foil with a focused ion beam (EMPA, Switzerland). The new pinhole P2 was placed $500 \mu \mathrm{m}$ upstream of the $\mathrm{KB}$ focal plane, with the sample now placed $300 \mu \mathrm{m}$ downstream of it. A ptychographic scan was then performed using $21 \times 21$ scan positions with a step size of $200 \mathrm{~nm}$ and an accumulation time of $1.5 \mathrm{~s}$ per scan point. Here the attenuation was set to $\sim 4 \times 10^{-3}$.

\section{Analysis}

The data were analyzed using the extended ptychographic iterative engine (ePIE) as outlined by Maiden \& Rodenburg (2009). For all datasets a region of interest of the $256 \times 256$ central pixels of the diffraction patterns was selected for reconstruction. With a Pilatus pixel width of $172 \mu \mathrm{m}$ this leads to a real-space pixel width of $19.6 \mathrm{~nm}$.

For all reconstructions the algorithm was run for 500 iterations, starting with a unit object amplitude and a Gaussian beam with a flat phase front as the probe. Remaining fluctuations in the reconstruction were eliminated by averaging over the last 50 iterations. The feedback parameters $\alpha$ and $\beta$ controlling the real-space update in the ePIE algorithm (Maiden \& Rodenburg, 2009) were set to a value of 0.35 . To prevent the reconstructed Fourier amplitude from growing without bound in non-sensitive regions of the Pilatus detector the amplitude value was limited here in each column of a horizontal non-sensitive stripe by the mean of the two lower and upper pixels adjacent to the non-sensitive column.

For Dataset 3 the probe reconstruction was found to contain a non-physical 'virtual point source' outside the physically meaningful probe region. This phenomenon can be explained by a large detector area with relatively low count rate (between 0 and 10 counts) in the high-spatial-frequency outer regions. If neither the sample nor the probe provides a strong constraint in the respective frequency region, a very 
small (less than five pixels in diameter) secondary source can obey the real- and Fourier-space constraints of the algorithm and thus be consistent with the data. Suppressing this artifact by introducing binary masks (Giewekemeyer et al., 2011a) on the probe in real space was not found to be successful here as it introduced more artifacts into the probe. As the relative contribution of the artificial point source to the total probe intensity is less than $1 \%$ in this case, no further constraints were introduced and the artifact was removed by subtraction after reconstruction.

\section{Results}

The reconstructed complex probe functions, propagated back to the focal plane of the KB system, are shown in Fig. 3. The focal plane was determined here by the maximum of a squared-intensity metric with respect to the position on the optical axis (Guizar-Sicairos, 2010; Wilke et al., 2012).

For all slit settings one obtains an almost flat phase distribution in the central amplitude maximum of the focus. When the focused beam is confined by a large pinhole of diameter $8 \mu \mathrm{m}$ (Dataset 1 and 2), rather prominent side lobes are visible in both the horizontal and vertical directions, originating from the rectangular confinement of the unfocused beam entering the KB system. Notably, these side lobes are well reconstructed, especially in the case of the smallest focus (Dataset 1), even though they extend over almost the full field of view. This effect, which is not expected given the insufficient sampling ratio for features extending over the full field of view, has been observed before for ptychographic reconstruction (for example, Giewekemeyer et al., 2011a) and may be connected to the strong over-determination of the reconstruction in ptychographic imaging. For Dataset 3, which was obtained with a much smaller beam-confining pinhole close to the focus (diameter $=1.4 \mu \mathrm{m}$ ), these side lobes are very efficiently suppressed. This results in a very effective lowpass filtering in the far field of the focus (see below).

As expected, one obtains an asymmetric focal shape for Dataset 1 where the vertical slits S1V were opened to

Figure 3

Figure 4
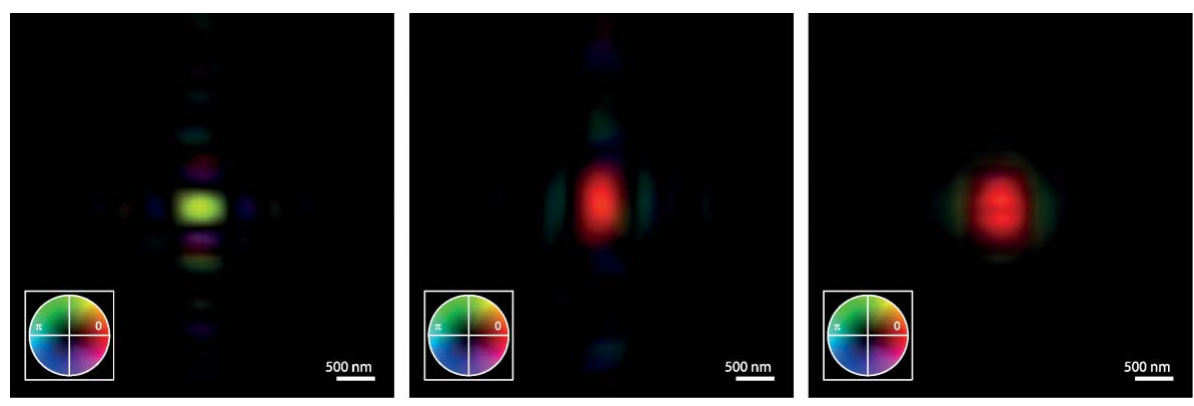

Reconstructed complex illumination functions, propagated to the numerically determined focal plane (see main text). The probe shown on the left corresponds to Dataset 1 , the one in the middle to Dataset 2, and the one on the right to Dataset 3.

$200 \mu \mathrm{m}$ while $\mathrm{S} 1 \mathrm{H}$ were kept at $100 \mu \mathrm{m}$. For S1V closed to $100 \mu \mathrm{m}$, the focus appears more symmetrical, even though it is still wider in the vertical than in the horizontal direction. This can be explained by the different focal distances of the two mirrors, leading to slightly different numerical apertures. Note that, as expected, the ratio of the focal lengths, $f_{\mathrm{V}} / f_{\mathrm{H}}=$ 1.51 , closely matches the ratio of the focal widths $(549 \mathrm{~nm} /$ $345 \mathrm{~nm} \simeq 1.591$ ).
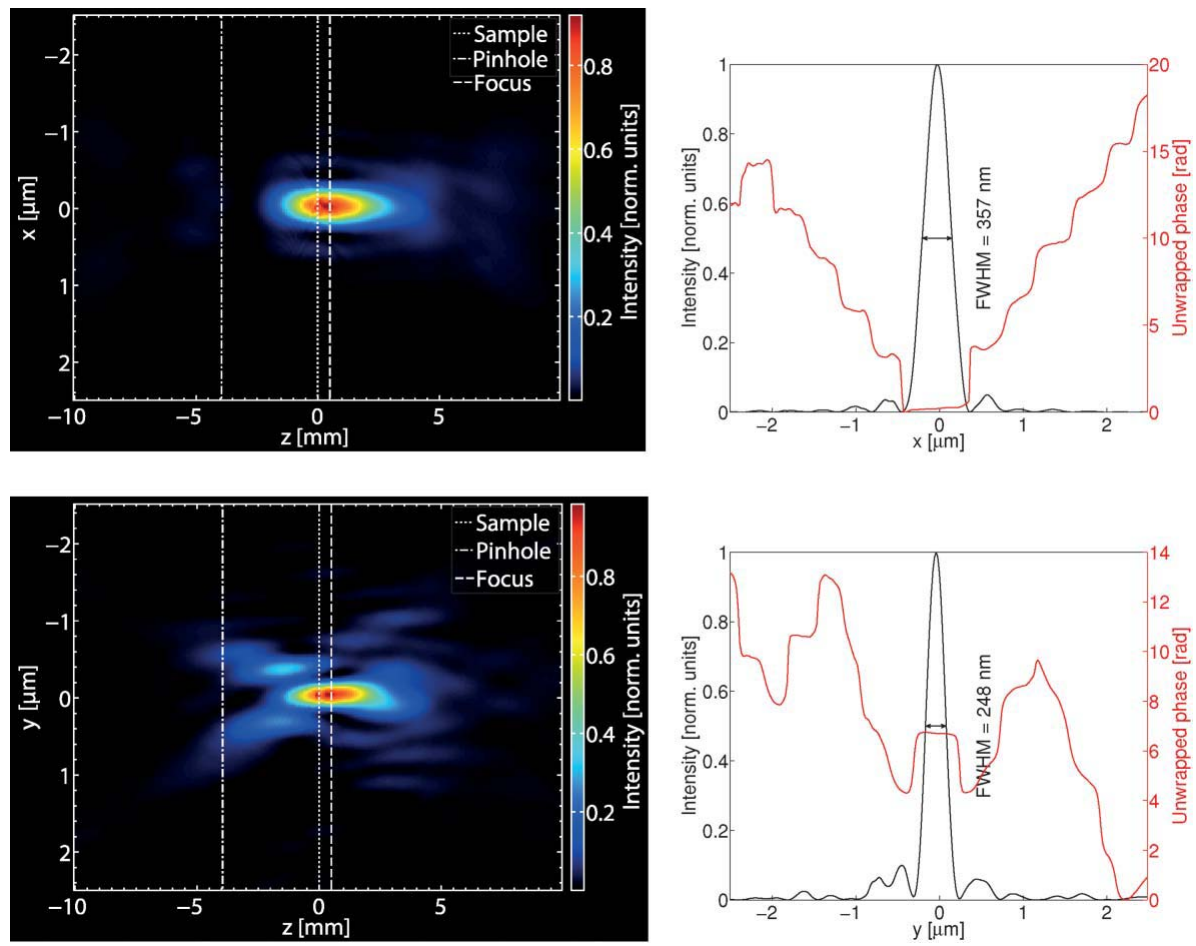

(Upper left and lower left) Horizontal and vertical slices through the reconstructed focal intensity (Dataset 1), obtained by numerical propagation of the reconstructed complex illumination $5 \mathrm{~mm}$ upstream and downstream of the reconstruction plane. The vertical slit S1V was opened here to $0.2 \mathrm{~mm}$, whereas the horizontal beam-defining slit $\mathrm{S} 1 \mathrm{H}$ was opened to $0.1 \mathrm{~mm}$, only half as wide. Accordingly, the focus is wider in the horizontal direction owing to the smaller numerical aperture. Note the indication of several distinct planes which correspond to the nominal plane of the pinhole P2 (see Fig. 1), the reconstruction, i.e. the sample plane, as well as the focal plane as determined by a numerical focusing criterion (see main text). (Upper right and lower right) The one-dimensional profiles of intensity (black) and phase (red) on the right correspond to the latter plane and indicate the asymmetry of the focus with respect to the horizontal and vertical directions. Both are selected such that they share the pixel of highest intensity in the two-dimensional intensity distribution. 

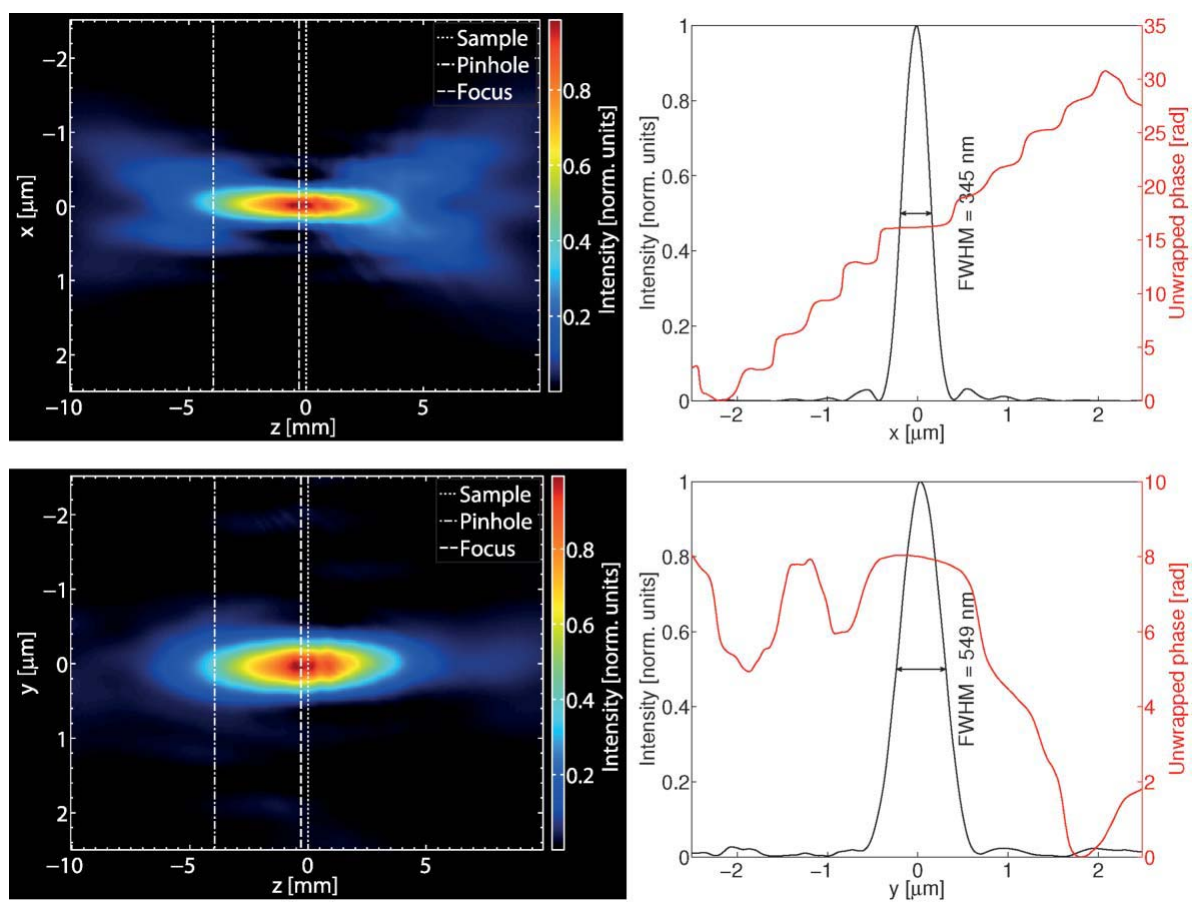

Figure 5

(Upper left and lower left) Horizontal and vertical slices through the reconstructed focal intensity (Dataset 2). The description of the different graphs is analogous to those of Fig. 4. The dataset was obtained with the same pinhole P2 as Dataset 1; however, the vertical slit S1V was closed here to $0.1 \mathrm{~mm}$, resulting in more symmetric one-dimensional intensity profiles (upper right and lower right).
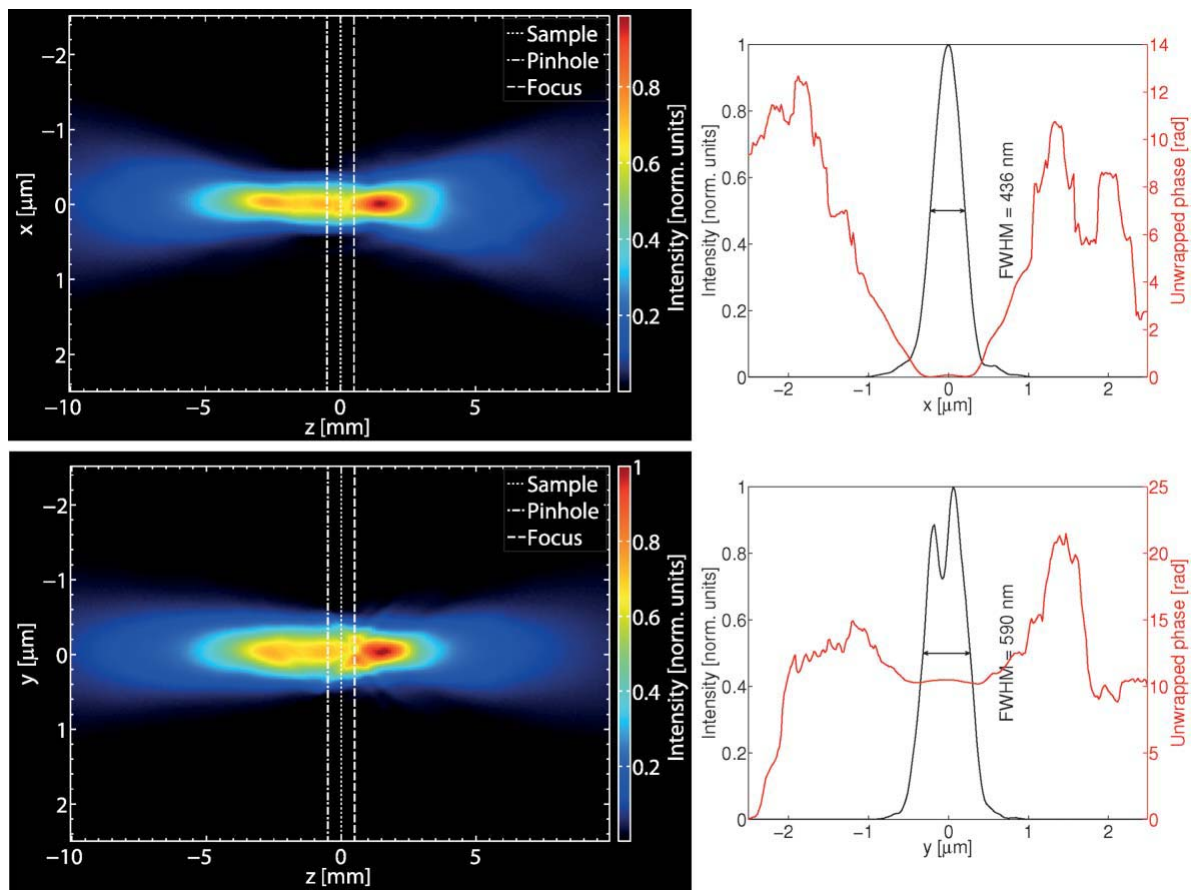

Figure 6

(Upper left and lower left) Horizontal and vertical slices through the reconstructed focal intensity (Dataset 3 ). In contrast to Datasets 1 and 2 here a smaller pinhole (diameter $=1.4 \mu \mathrm{m}$ ) has been used which significantly influences the focal intensity distribution. Here the beam-defining slits $\mathrm{S} 1 \mathrm{H}$ and S1V were set to $0.1 \mathrm{~mm}$. Graphs are analogous to those of Fig. 4. (Upper right and lower right) Even though in the numerically determined focal plane a double-focus is visible, the small pinhole P2 drastically reduces the side lobes in the focal plane. After further propagation to $\sim 1.6 \mathrm{~mm}$ downstream of the reconstruction plane the wavefield becomes a single focus in the horizontal and vertical directions and adopts almost a Gaussian shape (see Fig. 7).
Based on the total counts on the (photon-counting) detector and assuming the total intensity to be contained primarily within the central FWHM in the horizontal and vertical directions, one obtains the following intensity values (in photon numbers) in the focus: $4 \times 10^{6} \mu \mathrm{m}^{-2} \mathrm{~s}^{-1}$ for Dataset $1,2.4 \times 10^{4} \mu \mathrm{m}^{-2} \mathrm{~s}^{-1}$ for Dataset 2 and $1.3 \times 10^{5} \mu \mathrm{m}^{-2} \mathrm{~s}^{-1}$ for Dataset 3. Note that these focal intensities were limited by a conservative choice of the used attenuators, i.e. maximum countable flux per pixel in the detector, and may be different if an integrating detector with a higher dynamic range is used (Koerner et al., 2011).

In addition to the reconstructed complex amplitudes in the plane perpendicular to the optical axis, meridional $(y z)$ and sagittal $(x z)$ cuts through the propagated intensity near the focal plane are shown for all datasets in Figs. 4, 5 and 6. Note that for all of these graphs, in the area upstream of the pinhole $\mathrm{P} 2$, the back-propagated intensity no longer corresponds to the physical reality as the radiation absorbed by the pinhole does not contribute to the measured diffraction patterns and thus cannot be accounted for in the reconstruction. As expected, the depth-of-focus increases for a decreasing focal spot size. Furthermore, the one-dimensional lateral intensity and phase profiles along the horizontal $(x)$ and vertical $(y)$ coordinate directions are shown. The phase was unwrapped here using the standard Matlab (Matlab is a trademark of The Mathworks Inc.) one-dimensional unwrapping routine unwrap.m. Note that the phase is mathematically defined only up to integer multiples of $2 \pi$, so that corresponding side minima on opposing sides of the center can differ by approximately integer multiples of $2 \pi$. It can be observed that the phase is almost flat over the whole area of the central maximum for all focal spot sizes. As absolute phase values have no meaning, the profiles were shifted so that the minimum is zero. The expected general tendency of a constant phase in intensity maxima and highest gradients in intensity minima is well observable. In addition, it is clearly evident how the 
small pinhole near the focal plane suppresses any side minima in the focal plane ( $c f$. Fig. 6). On the other hand, unlike for Datasets 1 and 2, the small size of the pinhole here leads to an amplitude distortion in the focal plane. Notably, the focal plane as determined by the squared intensity metric is characterized by two local intensity maxima in the horizontal direction.

Upon further propagation of $1.5 \mathrm{~mm}$ from the focal plane, this double focus, however, turns into a single one that no longer has side lobes and is very reminiscent of an ideal Gaussian focus (see Fig. 7).

Fig. 8 shows the probe intensity in the detector plane, obtained by numerical forward propagation of the reconstructed complex wavefield in the sample plane. Note that closing the entrance slits $\mathrm{S} 1 \mathrm{H}$ and S1V corresponds directly to a smaller KB far-field pattern, as visible in Figs. 8(a) and 8(b). The features present in the far field for the second dataset with smaller slits (Fig. $8 b$ ) are reproduced in the central region of the far field for the first
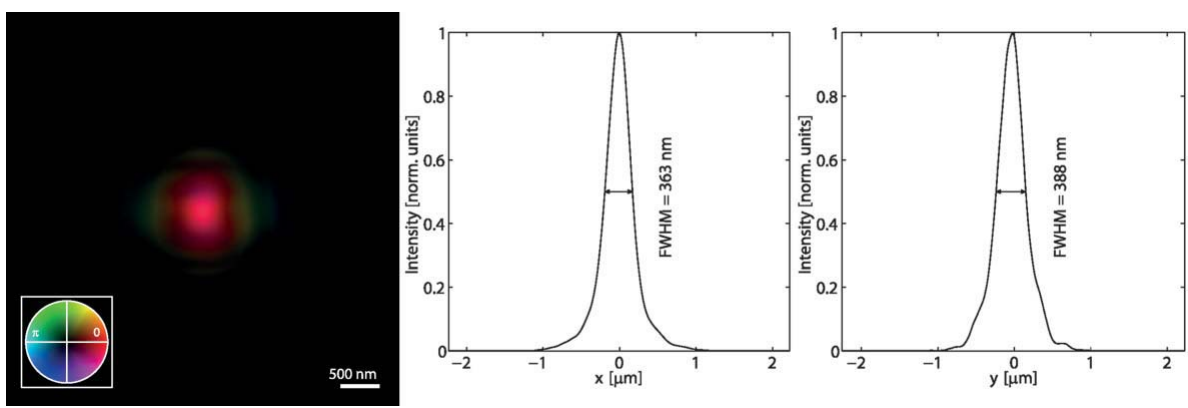

Figure 7

(Left) Reconstructed complex illumination function corresponding to Dataset 3, propagated $1.5 \mathrm{~mm}$ downstream of the reconstruction plane. The corresponding horizontal and vertical onedimensional profiles which share the pixel of highest intensity are shown in the middle and on the right. Note that the double focus in the vertical direction as visible in Fig. 6 has vanished.
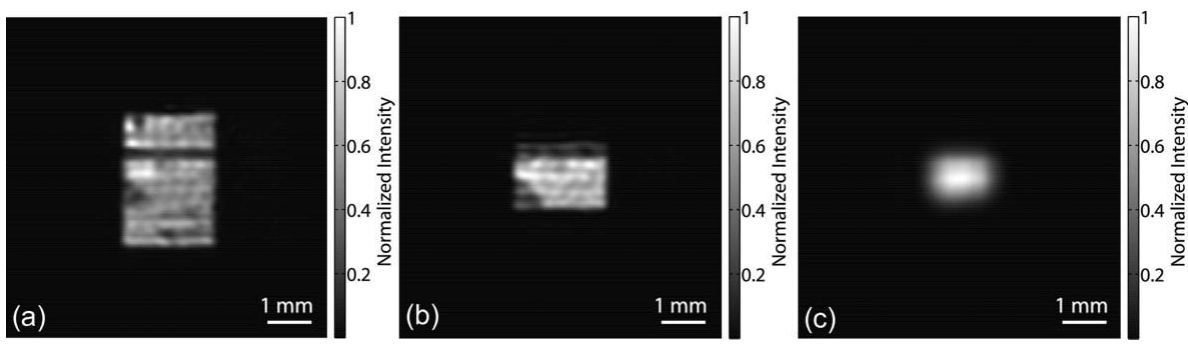

Figure 8

Reconstructed probe intensity, determined from the complex reconstructed probe, propagated to the detector plane, corresponding to Dataset $1(a)$, Dataset $2(b)$ and Dataset $3(c)$. Note that the far-field intensity distributions shown here have been calculated on a finer grid than given by the detector pixels to highlight the structure in the far-field patterns. dataset (Fig. 8a), obtained with larger

slit settings. The intensity fluctuations in the vertical direction can be attributed to height deviations in the vertically focusing mirror. In contrast, there are only comparably small artifacts along the horizontal direction, in accordance with smaller height deviations of the horizontally focusing mirror. Exchanging the large pinhole (diameter $=8 \mu \mathrm{m}$ ) with a small pinhole $($ diameter $=1.4 \mu \mathrm{m})$ upstream of the focus leads to a strong low-pass filtering effect on the KB far field. As a result, the two-dimensional intensity distribution in the detector plane has no internal substructure as is known from unfiltered KB beams. This makes the third optical configuration ideal for propagation-based coherent imaging of samples placed in between the KB focus and the detector (Cloetens et al., 1999; Olendrowitz et al., 2012; Krenkel et al., 2013). A clean far field without high-frequency intensity features is especially important here as the empty beam contributions to the diffraction pattern need to be separated from the sample signal, for example by normalization with the far field of the empty beam (Giewekemeyer et al., 2011b; Olendrowitz et al., 2012). In addition, the relatively large focal size which reduces scanning time makes it also very suitable for tomographic ptychography applied to biological specimens (Dierolf et al., 2010; Wilke et al., 2012).
Lastly, the complex object reconstructions corresponding to the three different datasets are shown in Fig. 9. For Datasets 1 and 2 small inaccuracies in the positioning of the piezo motor stages used for sample translations become apparent. Further refinement by iterative optimization of the motor positions is possible, for example by minimization of the standard experimental error metric (Guizar-Sicairos \& Fienup, 2008; Maiden et al., 2012). The reconstructed illumination function, however, is known to be rather robust against small positioning errors in ptychographic reconstructions (Schropp et al., 2010). As visible from the reconstructions, the resolution approaches the single-pixel value here, with the smallest stripe separation of $50 \mathrm{~nm}$ in the center of the Siemens star being very clearly resolved. This result has to be assessed in view of
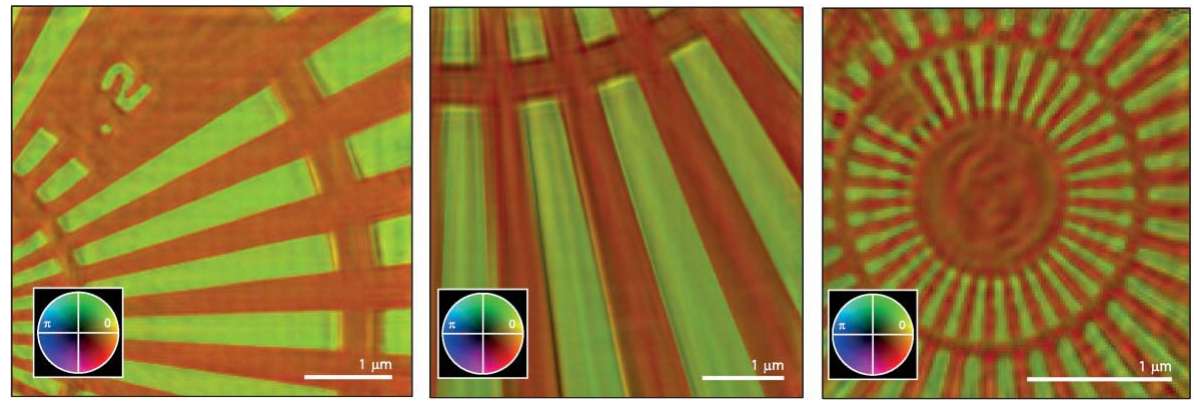

Figure 9

Reconstructed complex object functions, corresponding to Dataset 1 (left), Dataset 2 (middle) and Dataset 3 (right). 
the short measurement time (from 0.37 min for Dataset 1 to 11.03 min for Dataset 3, depending on absorbers that had to be introduced to protect the detector).

\section{Summary and conclusion}

In summary, we have studied the complex wavefield produced by a coherently illuminated KB optic under varying illumination conditions. In order to suppress side maxima which were attributed to refraction from the hybrid Ge/metal $\mathrm{KB}$ entrance slits, pinholes of different sizes were introduced upstream of the focal plane. This allowed for sufficient sampling of the coherent focus in its far field and thus for the application of ptychographic CDI. The possibility of adapting the focal intensity distribution to different possible experimental needs (high-resolution ptychographic CDI, propagation-based phase-contrast imaging) was shown by ptychographic reconstruction of focal distributions with varying slit settings and pinhole sizes. This underlies the versatility of the GINIX setup at the P10 coherence beamline which can be effectively used for ptychographic CDI of micrometer-scale objects with resolutions on the nanoscale or for in-line phasecontrast imaging of medium-sized samples (in the range of several hundred micrometers in diameter), and, in combination with waveguides, of smaller micrometer-scale specimens. The homogeneous KB far field without high-frequency artifacts that was obtained by using a small pinhole near the focal plane is especially important for the latter-mentioned propagation-based imaging methods.

The simple adjustment of the slit settings upstream of the KB was shown to provide a straightforward control of coherent focus sizes between 250 and $500 \mathrm{~nm}$, thus enabling a combination of scanning time, field of view and resolution that is optimized to the experimental need. At a fixed dwell time, one can thus either choose a large beam to cover a large sample area at the cost of obtainable resolution owing to a decrease in fluence, or one can select a small beam with high fluence to aim for high resolution at the cost of reducing the field of view.

Lastly, we note that the almost flat phase distribution that was observed in the center of all examined focal sizes also makes the setup suitable for plane-wave CDI. For this technique, however, especially when studying weakly scattering biological samples, the pinhole upstream of the sample should best be replaced by a soft corner (Shapiro et al., 2005) or guard slits (Takahashi et al., 2010) in order to limit non-sample scattering contributions to one quadrant of the detector.

It is our pleasure to thank Michael Sprung and the whole P10 beamline team for their outstanding and continuous support of the GINIX project and associated beam times during the past three years. Harry Quiney is thanked for discussions and advice. Financial support by the Collaborative Research Center 755 'Nanoscale Photonic Imaging' of the Deutsche Forschungsgemeinschaft and by the German Ministry of Science and Education under project number 05K10MGA is gratefully acknowledged.

\section{References}

Bartels, M., Priebe, M., Wilke, R. N., Krüger, S. P., Giewekemeyer, K., Kalbfleisch, S., Olendrowits, C., Sprung, M. \& Salditt, T. (2012). Opt. Nanosc. 1, 10.

Boutet, S. \& Williams, G. J. (2010). New J. Phys. 12, 035024.

Chapman, H. N., Barty, A., Marchesini, S., Noy, A., Hau-Riege, S. P., Cui, C., Howells, M. R., Rosen, R., He, H., Spence, J. C. H., Weierstall, U., Beetz, T., Jacobsen, C. \& Shapiro, D. (2006b). J. Opt. Soc. Am. A, 23, 1179-1200.

Chapman, H. N. et al. (2006a). Nat. Phys. 2, 839-843.

Chapman, H. N. et al. (2011). Nature (London), 470, 73-77.

Cloetens, P., Ludwig, W., Baruchel, J., Van Dyck, D., Van Landuyt, J., Guigay, J. P. \& Schlenker, M. (1999). Appl. Phys. Lett. 75, 2912 2914.

Dierolf, M., Menzel, A., Thibault, P., Schneider, P., Kewish, C. M., Wepf, R., Bunk, O. \& Pfeiffer, F. (2010). Nature (London), 467, 436-439.

Giewekemeyer, K., Beckers, M., Gorniak, T., Grunze, M., Salditt, T. \& Rosenhahn, A. (2011a). Opt. Express, 19, 1037-1050.

Giewekemeyer, K., Krüger, S. P., Kalbfleisch, S., Bartels, M., Beta, C. \& Salditt, T. (2011b). Phys. Rev. A, 83, 023804.

Guizar-Sicairos, M. (2010). PhD thesis, University of Rochester, USA.

Guizar-Sicairos, M., Evans-Lutterodt, K., Isakovic, A. F., Stein, A., Warren, J. B., Sandy, A. R., Narayanan, S. \& Fienup, J. R. (2010). Opt. Express, 18, 18374-18382.

Guizar-Sicairos, M. \& Fienup, J. R. (2008). Opt. Express, 16, 7264 7278.

Guizar-Sicairos, M., Holler, M., Diaz, A., Vila-Comamala, J., Bunk, O. \& Menzel, A. (2012). Phys. Rev. B, 86, 100103.

Guizar-Sicairos, M., Narayanan, S., Stein, A., Metzler, M., Sandy, A. R., Fienup, J. R. \& Evans-Lutterodt, K. (2011). Appl. Phys. Lett. 98, 111108 .

Harris, D. C. (2011). Proc. SPIE, 8016, 80160N.

Hignette, O., Cloetens, P., Rostaing, G., Bernard, P. \& Morawe, C. (2005). Rev. Sci. Instrum. 76, 063709.

Hönig, S., Hoppe, R., Patommel, J., Schropp, A., Stephan, S., Schöder, S., Burghammer, M. \& Schroer, C. G. (2011). Opt. Express, 19, 16324-16329.

Huang, X., Wojcik, M., Burdet, N., Peterson, I., Morrison, G. R., Vine, D. J., Legnini, D., Harder, R., Chu, Y. S. \& Robinson, I. K. (2012). Opt. Express, 20, 24038.

Kalbfleisch, S. (2012). PhD thesis, Georg-August-Universität Göttingen, Germany.

Kalbfleisch, S., Neubauer, H., Krüger, S. P., Bartels, M., Osterhoff, M., Mai, D. D., Giewekemeyer, K., Hartmann, B., Sprung, M. \& Salditt, T. (2011). AIP Conf. Proc. 1365, 96-99.

Kewish, C. M., Guizar-Sicairos, M., Liu, C., Qian, J., Shi, B., Benson, C., Khounsary, A. M., Vila-Comamala, J., Bunk, O., Fienup, J. R., Macrander, A. T. \& Assoufid, L. (2010a). Opt. Express, 18, 2342023427.

Kewish, C. M., Thibault, P., Dierolf, M., Bunk, O., Menzel, A., VilaComamala, J., Jefimovs, K. \& Pfeiffer, F. (2010b). Ultramicroscopy, 110, 325-329.

Koerner, L. J., Gillilan, R. E., Green, K. S., Wang, S. \& Gruner, S. M. (2011). J. Synchrotron Rad. 18, 148-156.

Krenkel, M., Bartels, M. \& Salditt, T. (2013). Opt. Express, 21, 2220 2235.

Krüger, S. P., Neubauer, H., Bartels, M., Kalbfleisch, S., Giewekemeyer, K., Wilbrandt, P. J., Sprung, M. \& Salditt, T. (2012). J. Synchrotron Rad. 19, 227-236.

Li, Y., Beck, R., Huang, T., Choi, M. C. \& Divinagracia, M. (2008). J. Appl. Cryst. 41, 1134-1139.

Maiden, A., Humphry, M., Sarahan, M., Kraus, B. \& Rodenburg, J. (2012). Ultramicroscopy, 120, 64-72.

Maiden, A. M. \& Rodenburg, J. M. (2009). Ultramicroscopy, 109, 1256-1262. 
Mastropietro, F., Carbone, D., Diaz, A., Eymery, J., Sentenac, A., Metzger, T. H., Chamard, V. \& Favre-Nicolin, V. (2011). Opt. Express, 19, 19223-19232.

Matsuyama, S., Mimura, H., Yumoto, H., Sano, Y., Yamamura, K., Yabashi, M., Nishino, Y., Tamasaku, K., Ishikawa, T. \& Yamauchi, K. (2006). Rev. Sci. Instrum. 77, 103102.

Miao, J., Charalambous, P., Kirz, J. \& Sayre, D. (1999). Nature (London), 400, 342-344.

Miao, J., Sayre, D. \& Chapman, H. N. (1998). J. Opt. Soc. Am. A, 15, 1662-1669.

Mimura, H., Handa, S., Kimura, T., Yumoto, H., Yamakawa, D., Yokoyama, H., Matsuyama, S., Inagaki, K., Yamamura, K., Sano, Y., Tamasaku, K., Nishino, Y., Yabashi, M., Ishikawa, T. \& Yamauchi, K. (2010). Nat. Phys. 6, 122-125.

Mimura, H., Morita, S., Kimura, T., Yamakawa, D., Lin, W., Uehara, Y., Matsuyama, S., Yumoto, H., Ohashi, H., Tamasaku, K., Nishino, Y., Yabashi, M., Ishikawa, T., Ohmori, H. \& Yamauchi, K. (2008). Rev. Sci. Instrum. 79, 083104.

Mimura, H., Yumoto, H., Matsuyama, S., Sano, Y., Yamamura, K., Mori, Y., Yabashi, M., Nishino, Y., Tamasaku, K., Ishikawa, T. \& Yamauchi, K. (2007). Appl. Phys. Lett. 90, 051903.

Nugent, K. A. (2010). Adv. Phys. 59, 1-99.

Olendrowitz, C., Bartels, M., Krenkel, M., Beerlink, A., Mokso, R., Sprung, M. \& Salditt, T. (2012). Phys. Med. Biol. 57, 5309-5323.

Osterhoff, M. (2011). PhD thesis, Georg-August-Universität, Göttingen, Germany.

Putkunz, C. T., Clark, J. N., Vine, D. J., Williams, G. J., Pfeifer, M. A., Balaur, E., McNulty, I., Nugent, K. A. \& Peele, A. G. (2011). Phys. Rev. Lett. 106, 013903.

Quiney, H. (2010). J. Mod. Opt. 57, 1109-1149.

Quiney, H. M., Nugent, K. A. \& Peele, A. G. (2005). Opt. Lett. 30, 1638-1640.

Robinson, I., Pfeiffer, F., Vartanyants, I., Sun, Y. \& Xia, Y. (2003). Opt. Express, 11, 2329-2334.

Rodenburg, J. (2008). Adv. Imaging Electron Phys. 150, 87-184.
Rodenburg, J. M., Hurst, A. C., Cullis, A. G., Dobson, B. R., Pfeiffer, F., Bunk, O., David, C., Jefimovs, K. \& Johnson, I. (2007). Phys. Rev. Lett. 98, 034801.

Sakdinawat, A. \& Attwood, D. (2010). Nat. Photon. 4, 840-848.

Salditt, T., Kalbfleisch, S., Osterhoff, M., Krüger, S. P., Bartels, M., Giewekemeyer, K., Neubauer, H. \& Sprung, M. (2011). Opt. Express, 19, 9656-9675.

Schropp, A., Boye, P., Feldkamp, J. M., Hoppe, R., Patommel, J., Samberg, D., Stephan, S., Giewekemeyer, K., Wilke, R. N., Salditt, T., Gulden, J., Mancuso, A. P., Vartanyants, I. A., Weckert, E., Schöder, S., Burghammer, M. \& Schroer, C. G. (2010). Appl. Phys. Lett. 96, 091102.

Seibert, M. M. et al. (2011). Nature (London), 470, 78-81.

Shapiro, D., Thibault, P., Beetz, T., Elser, V., Howells, M., Jacobsen, C., Kirz, J., Lima, E., Miao, H., Neiman, A. M. \& Sayre, D. (2005). Proc. Natl. Acad. Sci. USA, 102, 15343-15346.

Siewert, F., Buchheim, J., Boutet, S., Williams, G. J., Montanez, P. A., Krzywinski, J. \& Signorato, R. (2012). Opt. Express, 20, 4525-4536.

Takahashi, Y., Nishino, Y., Tsutsumi, R., Zettsu, N., Matsubara, E., Yamauchi, K. \& Ishikawa, T. (2010). Phys. Rev. B, 82, 214102.

Takahashi, Y., Suzuki, A., Zettsu, N., Kohmura, Y., Senba, Y., Ohashi, H., Yamauchi, K. \& Ishikawa, T. (2011). Phys. Rev. B, 83, 214109.

Thibault, P., Dierolf, M., Menzel, A., Bunk, O., David, C. \& Pfeiffer, F. (2008). Science, 321, 379-382.

Thibault, P. \& Elser, V. (2010). Annu. Rev. Condens. Matter Phys. 1, 237-255.

Toyokawa, H., Broennimann, C., Eikenberry, E., Henrich, B., Kawase, M., Kobas, M., Kraft, P., Sato, M., Schmitt, B., Suzuki, M., Tanida, H. \& Uruga, T. (2010). Nucl. Instrum. Methods Phys. Res. A, 623, 204-206.

Wilke, R. N., Priebe, M., Bartels, M., Giewekemeyer, K., Diaz, A., Karvinen, P. \& Salditt, T. (2012). Opt. Express, 20, 19232-19254.

Williams, G. J., Quiney, H. M., Dhal, B. B., Tran, C. Q., Nugent, K. A., Peele, A. G., Paterson, D. \& de Jonge, M. D. (2006). Phys. Rev. Lett. 97, 025506. 